\title{
Serum CD73 is a prognostic factor in patients with metastatic melanoma and is associated with response to anti- PD-1 therapy
}

\author{
Roberta Turiello, ${ }^{1,2}$ Mariaelena Capone, ${ }^{3}$ Diana Giannarelli, ${ }^{4}$ Elva Morretta, ${ }^{1}$ \\ Maria Chiara Monti, ${ }^{1}$ Gabriele Madonna, ${ }^{3}$ Domenico Mallardo, ${ }^{3}$ Lucia Festino, ${ }^{3}$ \\ Rosa Azzaro, ${ }^{3}$ Mitchell P Levesque, ${ }^{5}$ Laurence Imhof, ${ }^{5}$ Benjamin Weide, ${ }^{6}$ \\ Teresa Amaral, ${ }^{6}$ Marc Chevrier, ${ }^{7}$ Antje Sucker, ${ }^{8}$ Piotr Rutkowski, ${ }^{9}$ \\ Dirk Schadendorf, ${ }^{8}$ Celeste Lebbe, ${ }^{10}$ Jason John Luke (1),${ }^{11,12}$ \\ Kilian Wistuba-Hamprecht (10, ${ }^{6}$ Reinhard Dummer, ${ }^{5}$ Aldo Pinto, ${ }^{1}$ \\ Silvana Morello (D) , ${ }^{1}$ Paolo A Ascierto ${ }^{3}$
}

To cite: Turiello R, Capone M, Giannarelli D, et al. Serum CD73 is a prognostic factor in patients with metastatic melanoma and is associated with response to anti-PD-1 therapy. Journal for ImmunoTherapy of Cancer 2020;8:e001689. doi:10.1136/ jitc-2020-001689

RT and MC contributed equally.

Accepted 27 November 2020

Check for updates

(c) Author(s) (or their employer(s)) 2020. Re-use permitted under CC BY-NC. No commercial re-use. See rights and permissions. Published by BMJ.

For numbered affiliations see end of article.

Correspondence to Prof Silvana Morello; smorello@unisa.it

Dr Paolo A Ascierto; p.ascierto@istitutotumori.na.it

\section{ABSTRACT}

Background Inhibitors of immune checkpoint programmed cell death protein 1 (PD-1) receptor on $T$ cells have shown remarkable clinical outcomes in metastatic melanoma. However, most patients are resistant to therapy. Production of extracellular adenosine, via CD73-mediated catabolism of AMP, contributes to suppress T-cell-mediated responses against cancer. In this study, we analyzed the expression and activity of soluble CD73 in sera of patients with melanoma undergoing anti-PD-1 $1 \pm$ cytotoxic T-lymphocyte-associated antigen 4 therapy.

Methods Soluble CD73 expression and activity were retrospectively analyzed in serum of a total of 546 patients with melanoma from different centers before starting treatment (baseline) with anti-PD-1 agents, nivolumab or pembrolizumab, and compared with those of 96 healthy subjects. The CD73 activity was correlated with therapy response and survival of patients.

Results Patients with melanoma show significantly higher CD73 activity and expression than those observed in healthy donors $(p<0.0001)$. Elevated pretreatment levels of CD73 activity were associated with nonresponse to therapy with nivolumab or pembrolizumab. During treatment, levels of soluble CD73 activity remain unchanged from baseline and still stratify clinical responders from non-responders. High levels of serum CD73 enzymatic activity associate with reduced overall survival (OS; $\mathrm{HR}=1.36,95 \% \mathrm{Cl} 1.03$ to $1.78 ; p=0.03$ ) as well as progression-free survival (PFS; $H R=1.42,95 \%$ $\mathrm{Cl} 1.13$ to $1.79, \mathrm{p}=0.003)$. Further, the multivariate Cox regression analysis indicates that serum CD73 activity is an independent prognostic factor besides serum lactate dehydrogenase levels and the presence of brain metastases for both OS $(p=0.009)$ and PFS $(p=0.001)$. Conclusion Our data indicate the relevance of serum CD73 in patients with advanced melanoma receiving antiPD-1 therapy and support further investigation on targeting CD73 in combination with anti-PD-1 antibodies.

\section{INTRODUCTION}

Inhibitors of the programmed cell death protein 1 (PD-1)/programmed cell death ligand 1 (PD-L1) axis, as single agent or in combination with inhibitors of cytotoxic T-lymphocyte-associated antigen 4, can mediate tumor regression reaching clinically significant benefits. However, not all patients derive benefit from therapy with checkpoint inhibitors. ${ }^{1}{ }^{2}$ Therefore, a better understanding of the mechanisms that influence the therapeutic effectiveness of immune checkpoint inhibitors and that regulate the antitumor immunity is necessary. Identification of prognostic biomarkers to guide immunotherapeutic choices, and importantly, potential additional co-targets to increase the efficacy of anti-PD-1 therapy is also needed.

CD73 is an ecto-nucleotidase that exists in a membrane-bound form and in a soluble form, both able to hydrolyze extracellular AMP in a rate-limiting manner to generate extracellular adenosine. ${ }^{34}$ AMP as substrate for CD73 can derive from the hydrolysis of extracellular ATP and ADP through the CD39 or CD203a, or alternatively from the extracellular nicotinamide adenine dinucleotide (NAD)+ (via CD38/CD203a axis). ${ }^{5}$ CD73-derived adenosine is a key mediator of immunosuppression within tumor microenvironment, ${ }^{6}$ able to suppress the T-cell-mediated antitumor immunity, mainly through the stimulation of the A2A adenosine receptor subtype. ${ }^{7}$ In murine models of melanoma, breast cancer, colon cancer and prostate cancer, combined treatment with an anti-CD73 antibody and immune checkpoint inhibitors 
enhanced antitumor immune response and efficacy. ${ }^{8-10}$ Nowadays, monoclonal antibodies against CD73 represent the new generation of immune checkpoint inhibitors that are under clinical investigation in patients with cancer in combination with anti-PD-1 agents. ${ }^{11}$ Furthermore, emerging data show that overexpression of CD73 in human cancers or in peripheral blood of patients with cancer is often associated with poor survival, ${ }^{12}$ suggesting that CD73 may serve also as a prognostic biomarker in cancer.

The purpose of this study was to evaluate whether CD73 activity in sera of patients with melanoma correlates significantly with disease progression. Further, we intended to investigate whether the soluble CD73 activity also predicts response to therapy with pembrolizumab and nivolumab alone or in combination with the ipilimumab.

\section{METHODS}

\section{Patients and serum samples collection}

This retrospective multicenter study included a total of 546 patients with stage III or stage IV melanoma, treated with pembrolizumab, nivolumab or nivolumab in combination with ipilimumab. Patients received checkpoint immunotherapy in first, second or third line. Patient samples derived from six different centers: 100 patients from the Istituto Nazionale Tumori-IRCCS-Fondazione "G. Pascale", Naples (Italy), 53 patients from the University Hospital Essen (Germany), 111 patients from the University Hospital Tübingen (Germany), 88 patients from the Hospital Saint Louis of Paris (France), 144 patients from the University of Zurich (Switzerland) and 50 patients from the University of Chicago (USA). Most of the patients $(496 / 546,91 \%)$ were diagnosed with stage IV melanoma, according to VII edition of American Joint Committee on Cancer. ${ }^{13}$ Response to therapy was evaluated according to RECIST V.1.1 criteria. ${ }^{14}$ Tumor response was assessed every 12 weeks. Peripheral blood was obtained from all patients before (baseline) therapy started. Serum specimens were collected and stored at $-80^{\circ} \mathrm{C}$ until use. Serum samples from 41 patients with melanoma from the Istituto Nazionale Tumori-IRCCS—Fondazione "G. Pascale" in Naples and 19 patients from Chicago obtained during anti-PD-1 antibody therapy (3 months) were also available. Patients' characteristics, including sex, age, brain and bone metastasis, serum levels of the lactate dehydrogenase (LDH), status of BRAF mutation and line of therapy are summarized in table 1 . Serum samples of 96 healthy donors (69 males and 27 females), aged $>18$ years, were collected at Istituto Nazionale Tumori-IRCCS-Fondazione "G. Pascale", Naples, (Italy) and used as controls. The study was conducted in accordance with the provisions of the Declaration of Helsinki.

\section{AMPase activity}

AMPase activity was measured by a colorimetric assay based on the quantization of inorganic phosphate $(\mathrm{Pi})$, as previously described, ${ }^{15}$ in all serum samples from patients with melanoma and healthy subjects. Briefly, serum samples were thawed, and protein concentration was determined by Bradford assay. For each sample, $100 \mu \mathrm{g}$ of proteins were incubated for $10 \mathrm{~min}$ at $37^{\circ} \mathrm{C}$ in $100 \mu \mathrm{L}$ of reaction buffer $\left(10 \mathrm{mM} \mathrm{MgCl}_{2}, 120 \mathrm{mM} \mathrm{NaCl}, 5 \mathrm{mM} \mathrm{KCl}\right.$, $60 \mathrm{mM}$ glucose, $50 \mathrm{mM}$ Tris-HCl, pH 7.4; Sigma-Aldrich). AMP was used as substrate (2 mM; Sigma-Aldrich). The Pi levels produced during the AMP hydrolysis were measured by means of Malachite Green Phosphate Assay Kit (8118, ScienCell Research Laboratories). To quantify the baseline Pi levels, each sample was tested also without substrate (AMP). The net value of $\mathrm{Pi}$ was obtained by subtracting the baseline $\mathrm{Pi}$ amount from Pi released after AMP hydrolysis.

To confirm that the AMPase reaction was dependent on CD73 activity, 71 randomly chosen samples were also tested in the presence of adenosine 5'- $\alpha, \beta$-methylene diphosphate (APCP; $100 \mu \mathrm{M}$, Sigma-Aldrich), a potent chemical competitive inhibitor of CD73.

\section{Multiple reaction monitoring mass spectrometry}

The AMPase activity was also evaluated by means of multiple reaction monitoring mass spectrometry (MRMMS) in randomly chosen samples of patients with melanoma and healthy donors. Sample volumes containing $100 \mu \mathrm{g}$ of proteins were equilibrated in the reaction buffer, as described above, at $37^{\circ} \mathrm{C}$ for $10 \mathrm{~min}$; then samples were treated or not with the APCP inhibitor $(100 \mu \mathrm{M})$ or with the anti-human CD73 mAb (Clone 7G2) (5 $\mu \mathrm{g} / \mathrm{mL}$ ) that inhibits the CD73 enzymatic activity as previously described ${ }^{16}$ for $30 \mathrm{~min}$ at $37^{\circ} \mathrm{C}$. Then, ${ }^{15} \mathrm{~N}$-AMP $(25 \mu \mathrm{M}$; Sigma-Aldrich) was added and the reaction proceeded for $120 \mathrm{~min}$ at $37^{\circ} \mathrm{C}$. Finally, $25 \mu \mathrm{L}$ of each sample were treated with Trichloroacetic acid (TCA; $5 \%$ final concentration; Sigma-Aldrich) to quench the reaction and then centrifuged $\left(3000 \mathrm{~g}, 10 \mathrm{~min}\right.$ at $\left.4^{\circ} \mathrm{C}\right)$. The supernatants were dried and dissolved in $50 \mu \mathrm{L}$ of $10 \mathrm{mM}$ Ammonium Acetate (AmAC, Sigma-Aldrich) containing $0.1 \%$ acetic acid (AA; Sigma-Aldrich). The obtained samples were submitted to ultra-performance liquid chromatographyelectrospray ionization (UPLC-ESI)-MRM-MS analysis to quantify the ${ }^{15} \mathrm{~N}$-AMP. UPLC-ESI-MRM-MS analyses were performed on a 6500 Q-TRAP from AB Sciex equipped with Shimadzu LC-20A and Autosampler systems, injecting $2 \mu \mathrm{L}$ of each sample. UPLC separation was performed on a Luna Omega Polar $1.6 \mu \mathrm{m}$ C18 100 Å column $(50 \times 2.10$ $\mathrm{mm}$, Phenomenex, Torrance, California, USA) at a flow rate of $400 \mu \mathrm{L} / \mathrm{min} ; 10 \mathrm{mM} \mathrm{AmAc} / 0.1 \% \mathrm{AA}$ in $\mathrm{H}_{2} \mathrm{O}$ (phase A) and $0.1 \% \mathrm{AA}$ in $\mathrm{MeOH}$ (phase $\mathrm{B}$ ) were used as mobile phases and the following gradient was exploited: $0 \% \mathrm{~B}$ from 0 to $0.5 \mathrm{~min}, 0 \%$ to $5 \% \mathrm{~B}$ over $2.5 \mathrm{~min}, 5 \%$ to $95 \% \mathrm{~B}$ over $2 \mathrm{~min}$, then held at $95 \% \mathrm{~B}$ for $2 \mathrm{~min}$ and re-equilibrated to $0 \% \mathrm{~B}$ over $5 \mathrm{~min}$. Q-TRAP 6500 was operated in positive MRM scanning mode, with declustering potential set at $80 \mathrm{~V}$, entrance potential at $10 \mathrm{~V}$, collision energy at $30 \mathrm{~V}$ and cell exit potential at $12 \mathrm{~V}$. 
Table 1 Clinicopathological characteristics of patients with melanoma

\begin{tabular}{|c|c|c|c|c|c|c|}
\hline \multirow[b]{2}{*}{ Total } & \multicolumn{2}{|c|}{$\begin{array}{l}\text { Nivolumab or pembrolizumab } \\
\text { therapy } \\
\text { (no, \%) }\end{array}$} & \multicolumn{2}{|c|}{$\begin{array}{l}\text { Nivolumab+ipilimumab } \\
\text { therapy } \\
\text { (no, \%) }\end{array}$} & \multicolumn{2}{|c|}{$\begin{array}{l}\text { Total } \\
\text { (no, \%) }\end{array}$} \\
\hline & 453 & 100 & 93 & 100 & 546 & 100 \\
\hline $\begin{array}{l}\text { Median age, years } \\
\text { (range, 19-94) }\end{array}$ & 65 & & 61 & & 64.5 & \\
\hline \multicolumn{7}{|l|}{ Sex } \\
\hline Male & 271 & 59.8 & 53 & 57.0 & 324 & 59.3 \\
\hline Female & 182 & 40.2 & 40 & 43.0 & 222 & 40.7 \\
\hline \multicolumn{7}{|l|}{ Disease stage } \\
\hline III & 48 & 10.6 & 4 & 4.3 & 52 & 9.5 \\
\hline IV & 402 & 88.7 & 89 & 95.7 & 491 & 89.9 \\
\hline Unknown & 3 & 0.66 & 0 & & 3 & 0.5 \\
\hline \multicolumn{7}{|c|}{ Serum lactate dehydrogenase } \\
\hline$<U L N$ & 308 & 74.0 & 51 & 54.8 & 359 & 65.8 \\
\hline$\geq$ ULN & 116 & 19.6 & 41 & 44.1 & 157 & 28.7 \\
\hline Unknown & 29 & 6.4 & 1 & 1.1 & 30 & 5.5 \\
\hline \multicolumn{7}{|l|}{ BRAF status } \\
\hline wt & 270 & 59.6 & 45 & 48.4 & 315 & 57.7 \\
\hline mut & 147 & 32.5 & 33 & 35.5 & 180 & 33.0 \\
\hline Unknown & 36 & 7.9 & 15 & 16.1 & 51 & 9.3 \\
\hline \multicolumn{7}{|l|}{ Brain metastasis } \\
\hline Yes & 78 & 17.2 & 9 & 9.7 & 87 & 15.9 \\
\hline No & 307 & 67.8 & 41 & 44.1 & 348 & 63.7 \\
\hline Unknown & 68 & 15.0 & 43 & 46.2 & 111 & 20.3 \\
\hline \multicolumn{7}{|l|}{ Bone metastasis } \\
\hline Yes & 80 & 17.6 & 13 & 14.0 & 93 & 17.0 \\
\hline No & 302 & 66.7 & 37 & 39.8 & 339 & 62.1 \\
\hline Unknown & 71 & 15.7 & 43 & 46.2 & 114 & 20.9 \\
\hline \multicolumn{7}{|l|}{ Line of treatment } \\
\hline 1 & 218 & 48.1 & 38 & 40.9 & 256 & 46.9 \\
\hline 2 & 134 & 29.6 & 27 & 29.0 & 161 & 29.4 \\
\hline$\geq 3$ & 101 & 22.3 & 28 & 30.1 & 129 & 23.6 \\
\hline
\end{tabular}

mut, mutated; ULN, upper limit of normal; wt, wild-type.

${ }^{15} \mathrm{~N}-\mathrm{AMP}$ was monitored through the 353.19/140.00 transition: the area of its related peak in each sample was measured using the Analyst Software from AB Sciex.

\section{Enzyme-linked immunosorbent assay}

CD73 was quantified in randomly selected sera of 83 patients with melanoma and 38 healthy subjects, using a commercially available ELISA kit (ab213761, Abcam), following the manufacturer's instructions.

\section{Statistical analyses}

Results are presented using absolute frequencies and percentages when referring to categorical variables, and median and range when considering quantitative variables. Survival time was analyzed with the KaplanMeier method, and log-rank test was used to test for differences. Proportional hazard models were used to estimate HRs and their 95\% CIs. Multivariate analysis was performed using a forward stepwise method based on Wald statistics with $p=0.05$ and $p=0.10$ as enter and remove limit. IBM SPSS V.21.0 statistical software was used for these analyses.

All other analyses were performed using GraphPad Prism V.7.0. In figures, mean \pm SD is shown. The MannWhitney test or analysis of variance test were used for two groups comparison or three or more groups comparison, respectively. The number of samples for each subgroup is indicated in the figure legends. 
For all the analyses, statistical significance was set at $\mathrm{p}<0.05$.

\section{RESULTS}

Clinicopathological characteristics of patients with melanoma

A total of 546 patients with stage III/IV melanoma were included in this study (table 1). The median age was 65 years old; 324 patients $(59.3 \%)$ were male and 222 $(40.7 \%)$ were female. One hundred eighty patients had a $B R A F$ mutation and 315 patients were $B R A F$ wild-type; in 51 patients, the $B R A F$ mutational status was unknown. Brain metastases were present in $87 / 435$ patients, while bone metastases were present in 93/432. Regarding the treatment regimens, $83 \%$ of the patients received PD-1 monotherapy with nivolumab $(\mathrm{n}=182)$ or pembrolizumab $(\mathrm{n}=271)$ and $17 \%$ received nivolumab plus ipilimumab $(\mathrm{n}=93)$. As for the treatment line, 218 patients received first-line PD-1 monotherapy, 134 patients received second-line PD-1 monotherapy and 101 patients received PD-1 monotherapy in third line or later. Thirty-eight patients received first-line nivolumab plus ipilimumab, 27 patients second line and 28 patients third line or later. The baseline clinicopathological characteristics of all patients, grouped according to the treatment regimen, are summarized in table 1 . The baseline characteristics of the patients' groups were comparable (table 1). Details on patients' characteristics of the single center are given in online supplemental table 1 . The median progressionfree survival (PFS) was 7.1 months (95\% CI: 5.3 to 8.8) and the median overall survival (OS) was 27.0 months (95\% CI: 22.7 to 31.3 ).

Excluding patients who were lost to follow-up, in the group of patients receiving anti-PD-1 agents in monotherapy $(n=447)$, a total of 154 patients $(35 \%)$ had a complete response $(\mathrm{CR}, \mathrm{n}=87$ ) or partial response (PR, $\mathrm{n}=67)$, while the others $293(65 \%)$ showed progressive disease $(\mathrm{PD}, \mathrm{n}=205)$ or stable disease $(\mathrm{SD}, \mathrm{n}=88)$. In the subgroup of patients receiving nivolumab plus ipilimumab $(n=90), 28$ patients $(32 \%)$ presented $C R(n=3)$ or PR $(n=25)$, while 62 patients $(68 \%)$ had SD $(n=16)$ or PD $(n=46)$. The median follow-up for patients receiving nivolumab or pembrolizumab in monotherapy was 29 months; for patients receiving nivolumab in combination with ipilimumab, the median follow-up was 22 months.

\section{Baseline CD73 activity and expression are high in serum of patients with melanoma}

We evaluated the AMP hydrolysis in serum samples of patients with melanoma before starting treatment with anti-PD-1 agents (baseline) and in healthy donors. The AMPase activity was significantly higher in patients with melanoma (37.85 $\pm 44.31 \mathrm{pmol} / \mathrm{min} / \mathrm{mg}$ protein) than in healthy donors $(7.88 \pm 15.5 \mathrm{pmol} / \mathrm{min} / \mathrm{mg}$ protein) $(\mathrm{p}<0.0001)$ (figure 1A). In melanoma group, 208 patients presented an AMPase activity higher than mean value $37.85 \mathrm{pmol} / \mathrm{min} / \mathrm{mg}$ protein. To evaluate if $\mathrm{CD} 73$ was responsible for AMP hydrolysis, samples from patients

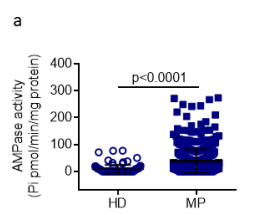

b
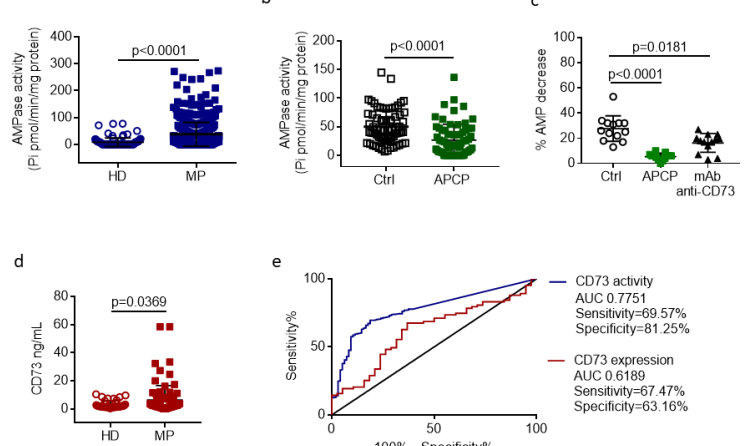

Figure 1 The level of CD73 enzymatic activity in serum discriminates patients with metastatic melanoma from healthy donors. A, AMPase activity in serum samples from patients with melanoma (MP, $n=546$ ) and healthy donors (HD; $n=96)$, measured with malachite green assay kit. $B$, The AMP hydrolysis in samples of MPs is significantly reduced in presence of the selective CD73 inhibitor, APCP $(100 \mu \mathrm{M})$ $(n=71)$. C, Percentage of hydrolysis of ${ }^{15} \mathrm{~N}$ AMP by multiple reaction monitoring mass spectrometry in samples of MPs $(n=13)$ in presence of APCP $(100 \mu M)$ or anti-human CD73 monoclonal antibody (clone $7 \mathrm{G} 2)(5 \mu \mathrm{g} / \mathrm{mL}$ ) or vehicle (Ctrl). $D$, ELISA of CD73 in serum of MPs $(n=83)$ and HD $(n=38)$. E, Receiving operating characteristic curve analysis for CD73 protein expression and CD73 enzymatic activity in MPs compared with healthy donors. Mean $\pm S D$ is shown. $P$ values are from two-sided Mann-Whitney test $(A, B, D)$ or oneway analysis of variance test (C). APCP, $5^{\prime}$ - $\alpha, \beta$-methylene diphosphate; $A \cup C$, area under the curve; $\mathrm{Pi}$, inorganic phosphate.

were preincubated with the most potent known CD73 inhibitor, APCP, resulting in a marked reduction in the levels of $\mathrm{Pi}(\mathrm{p}<0.0001)$ (figure $1 \mathrm{~B})$. To find out whether alkaline phosphatases might contribute to dephosphorylate AMP into adenosine and $\mathrm{Pi}^{4}{ }^{4}$ we evaluated the activity of tissue-non-specific alkaline phosphatase present in the bloodstream in a soluble form. ${ }^{17}$ The activity of these enzymes, evaluated by measuring the hydrolysis of the substrate bis(p-nitrophenyl)phosphate, was observed only at pH 9.8 (online supplemental figure 1), which is the optimal condition for their catalytic activity, ${ }^{18}$ but not at physiological $\mathrm{pH} 7.4$ (online supplemental figure 1). These results suggest that non-specific phosphatases do not contribute to hydrolyze the substrate AMP at the experimental conditions we measured the CD73 activity. To check further if the AMPase activity was dependent on CD73, we evaluated the reduction of ${ }^{15} \mathrm{~N}$ AMP as substrate by MRM-MS in presence and absence of APCP or anti-human CD73 monoclonal antibody (Clone 7G2) in a subset of patients with melanoma. The percentage of ${ }^{15} \mathrm{~N}$ AMP decrease in samples from patients with melanoma was significantly reduced in presence of the $\mathrm{CD} 73$ inhibitor APCP as well as in presence of the specific antiCD73 antibody compared with control (figure 1C). In healthy donors' serum samples, the percentage of ${ }^{15} \mathrm{~N}$ AMP hydrolysis was very low or nearly absent (data not shown). Altogether these results indicate that exogenous 
AMP hydrolysis in serum of patients with melanoma is dependent on CD73.

The expression of CD73 was determined by ELISA in randomly chosen serum samples. The expression level of serum CD73 was significantly higher in patients with melanoma than in healthy donors $(\mathrm{p}=0.0369)$ (figure 1d). Of 83 patients with melanoma tested for CD73 expression, 21 patients presented a concentration of CD73 higher than mean value $6.30 \mathrm{ng} / \mathrm{mL}$.

The analysis of the results and the receiving operating characteristic curve for CD73 activity and CD73 expression indicate that the levels of $\mathrm{CD} 73$ activity best stratified patients with melanoma from healthy subjects (figure 1e).

CD73 is the key enzyme in the generation of extracellular adenosine from AMP. AMP is in turn produced by ADP/ATP through the enzymatic activity of the ectonucleotidase CD39. Therefore, both CD39 and CD73, in sequential reactions, are critically involved in the regulation of extracellular adenosine levels in solid tumors. ${ }^{6}{ }^{11}$ At this regard, we also evaluated the ATPase activity in serum samples of patients with melanoma using ATP as substrate. We observed that the percentage of ATP reduction in serum samples of patients $(n=26)$, also in presence of the CD39 inhibitor ARL 67156, was negligible (online supplemental figure $2 \mathrm{a}$ ). These results rule out any relevant CD39 activity in serum of patients with melanoma, according to previous reports showing that ATPase and ADPase activities in human blood are mainly mediated by cell-bound enzymes. ${ }^{19}$ Expression of CD39 was also evaluated in serum samples from healthy subjects $(n=10)$ and patients with melanoma $(n=31)$. No difference was observed in CD39 expression between the two groups (online supplemental figure 2b).

\section{CD73 activity levels correlate with response to therapy}

We have previously reported that the serum CD73 activity in patients with melanoma correlates with response to nivolumab treatment. ${ }^{20}$ Here, we have confirmed this finding in a larger number of patients from different centers, who received pembrolizumab, or nivolumab alone or in combination with ipilimumab. The pretreatment activity of CD73 was significantly higher in patients with PD or SD compared with patients with CR or PR $(\mathrm{p}<0.0001$; figure $2 \mathrm{a})$. A detailed analysis of CD73 relative to patients treated with nivolumab or pembrolizumab alone or with nivolumab plus ipilimumab was performed. The baseline CD73 activity in patients with melanoma treated with nivolumab or pembrolizumab alone was significantly increased in patients with PD or SD compared with patients with CR or PR ( $p=0.0013$; figure $2 b)$. The baseline CD73 activity was significantly different also between patients who respond $(\mathrm{CR} / \mathrm{PR})$ or failed $(\mathrm{SD} /$ $\mathrm{PD})$ to the combined treatment nivolumab plus ipilimumab ( $\mathrm{p}=0.0052$; figure $2 \mathrm{c}$ ). In addition, the pretreatment levels of CD73 activity were significantly higher in SD/PD compared with $\mathrm{CR} / \mathrm{PR}$ patients who have received first anti-PD-1 monotherapy (online supplemental figure 3). CR/PR patients who have received anti-PD-1 second-line
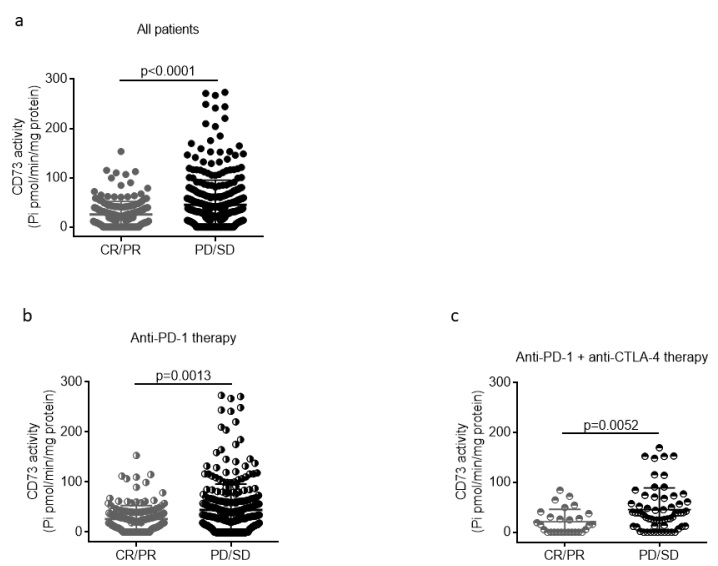

Figure 2 The baseline serum activity of CD73 predicts response to anti-PD-1 therapy. A, Baseline CD73 activity in all patients, receiving anti-PD-1 agents alone $(n=447)$ or in combination with anti-CTLA-4 $(n=90)$, with clinical response (complete response (CR)/partial response $(\mathrm{PR})$; $n=183$ ) or without clinical response (progressive disease (PD)/stable disease (SD); $n=354$ ). B, Baseline CD73 activity in patients with or without clinical response to nivolumab or pembrolizumab alone (CR/PR, $n=154$; PD/SD, $n=293$ ), or to nivolumab plus ipilimumab (CR/PR, $n=28$; PD/SD, $n=62$ ) (C). Mean $\pm S D$ is shown. $P$ values are from two-sided MannWhitney test. CTLA-4, cytotoxic T-lymphocyte-associated antigen 4; PD-1, programmed cell death protein 1; Pi, inorganic phosphate.

treatment showed a tendency toward lower CD73 activity compared with SD/PD patients but it does not reach significance (online supplemental figure 3 ).

Next, we evaluated the activity of serum CD73 in patients receiving anti-PD-1 therapy within 3 months of therapy. Changes in CD73 activity were not evident at month 3 compared with baseline when the entire group of patients was evaluated (figure $3 \mathrm{~A}$ ). Notably, in patients who did not respond to therapy with nivolumab or pembrolizumab, the levels of CD73 activity remained higher than those with clinical response at month 3 of treatment and unchanged from baseline (figure 3B). Analyses were not performed in nivolumab plus ipilimumab group of patients on treatment due to the small number of available samples.

\section{Associations of baseline CD73 activity with survival}

Because baseline CD73 enzymatic activity correlates with the outcome of anti-PD-1 therapy, we next investigated the associations between the enzymatic activity of CD73 and OS. The discriminative cutoff point of CD73 for early progression was set at 2 standard deviation (SD) from the mean of CD73 activity in healthy subjects (HD CD73 activity $7.88 \pm 15.5 \mathrm{pmol} / \mathrm{min} / \mathrm{mg}$ protein): 38.8 $\mathrm{pmol} / \mathrm{min} / \mathrm{mg}$ protein. We found that high levels of serum CD73 enzymatic activity associate with worse OS ( $\mathrm{HR}=1.36$; 95\% CI 1.03 to $1.78 ; \mathrm{p}=0.03$ ) (figure $4 \mathrm{a}$ ) and PFS (HR=1.42; 95\% CI 1.13 to 1.79 ; $\mathrm{p}=0.003$ ) in patients treated with nivolumab or pembrolizumab alone. In these latter group receiving anti-PD-1 agent as first line, we also observed that the median PFS was significantly 


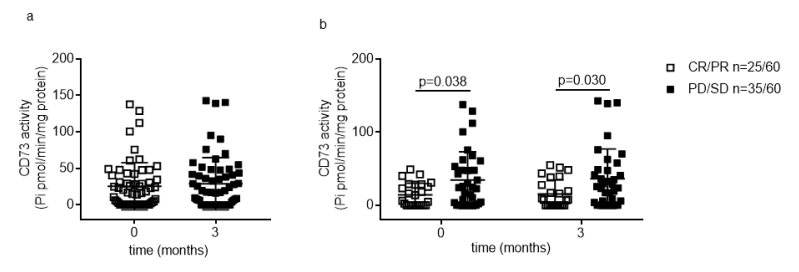

Figure 3 Levels of serum CD73 activity within 3 months therapy with anti-PD-1 agents. A, Levels of serum CD73 activity at time points pretreatment $(0)$ and on-treatment ( 3 months) in all patients analyzed $(n=60)$. B, Comparison of serum CD73 activity in patients with or without clinical benefits to nivolumab or pembrolizumab at time points pretreatment $(0)$ and on-treatment ( 3 months). Mean $\pm S D$ is shown. $P$ values were calculated with the two-way analysis of variance (B). CR,complete response; PD-1, programmed cell death protein 1; PD, progressive disease; $\mathrm{Pi}$, inorganic phosphate; PR, partial response; SD, stable disease.

longer in patients with low CD73 activity (12.3 months, 95\% CI 6.6 to 17.9 ) than patients with high CD73 activity (4.2 months, 95\% CI 2.4 to 6.0$)(\mathrm{p}<0.001)$, but not in patients treated with anti-PD-1 agent as second line $(\mathrm{p}=0.17)$ (online supplemental figure $4 \mathrm{ab}$ ), respectively). Regarding the OS, patients treated with anti-PD-1 agent as first line with low CD73 activity showed a tendency to a longer survival (27.9 months, $95 \%$ CI 22.5 to 33.2) than patients with high CD73 activity (18.4 months, 95\% CI 0.4 to 36.4$)(\mathrm{p}=0.09)$; in patients receiving anti-PD-1 treatment as second line, no significant differences in OS were observed $(\mathrm{p}=0.19)$ (online supplemental figure $4 \mathrm{~cd}$, respectively).

No association was found for OS (HR 1.41; 95\% CI 0.82 to $2.44 ; \mathrm{p}=0.23$; figure $4 \mathrm{~b}$ ) or PFS (HR $1.31 ; 95 \%$ CI 0.82 to $2.08 ; \mathrm{p}=0.26$ ) in patients receiving nivolumab plus ipilimumab.

Among patients treated with nivolumab or pembrolizumab alone, additional correlations of age, sex, LDH levels, brain or bone metastases and BRAF mutation status with OS were also performed. In patients aged $<65$ years, we found a negative correlation with OS for high

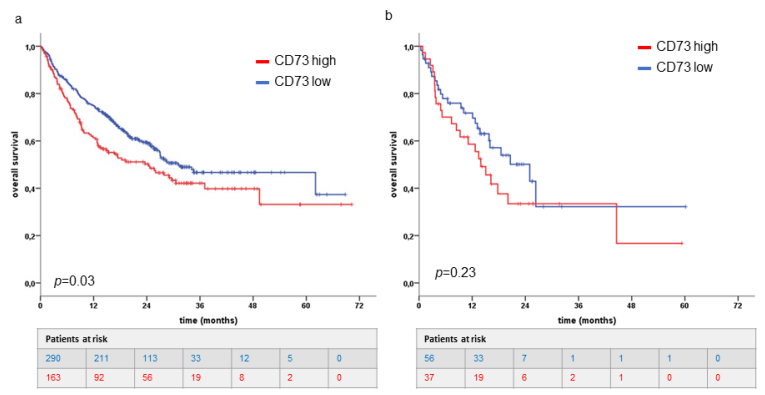

Figure 4 Prognostic value of soluble CD73 in patients with metastatic melanoma. A, Kaplan-Meier curves estimate overall survival of all patients receiving nivolumab or pembrolizumab or (B) nivolumab plus ipilimumab according to baseline serum activity of CD73. CD73 high (red line) is $>38.8 \mathrm{pmol} / \mathrm{min} / \mathrm{mg}$ protein and CD73 low is $<38.8 \mathrm{pmol} / \mathrm{min} /$ $\mathrm{mg}$ protein (blue line). $\mathrm{P}$ values were calculated using the logrank test.

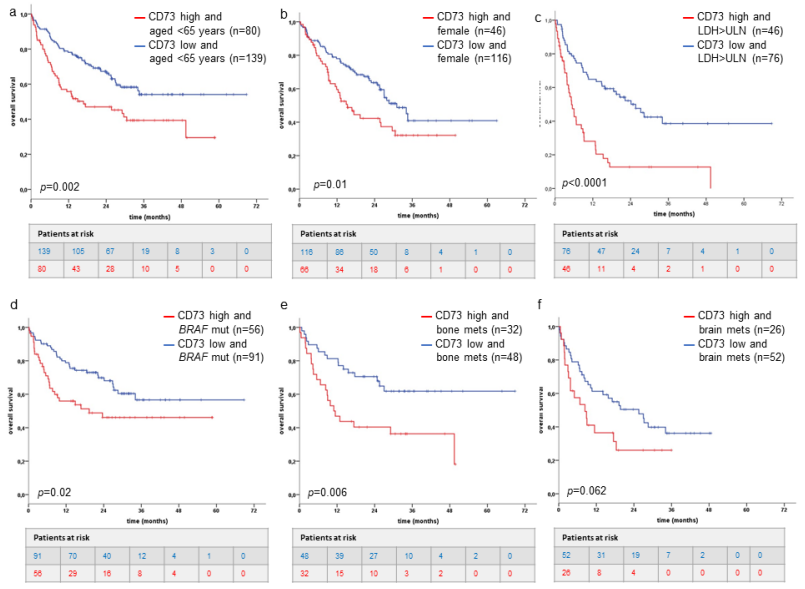

Figure 5 Prognostic value of soluble CD73 in subgroups of patients with metastatic melanoma receiving anti-PD-1 therapy alone. Groups of patients aged $<65$ years (A) or female (B) or with elevated serum lactate dehydrogenase $(\mathrm{LDH})$ levels $(C)$ or with BRAF mutations $(\mathrm{D})$ or with bone metastases (E) or with brain metastases (F) and high CD73 activity (>38.8 $\mathrm{pmol} / \mathrm{min} / \mathrm{mg}$ protein) (red line) have worse overall survival compared with patients with low CD73 activity ( $<38.8 \mathrm{pmol} / \mathrm{min} / \mathrm{mg}$ protein). P values were calculated using the log-rank test. ULN, upper limit of normal.

levels of CD73 activity compared with patients with low CD73 activity (HR=1.87; 95\% CI 1.26 to 2.78 ; $\mathrm{p}=0.002$ ) (figure 5a) but not in elderly patients (data not shown). A negative correlation with OS for high levels of CD73 activity was also observed in the female patients' group ( $\mathrm{HR}=1.69 ; 95 \%$ CI 1.11 to $2.59 ; \mathrm{p}=0.01$ ) (figure $5 \mathrm{~b}$ ) but not in the male patients' group (data not shown). Among patients with elevated LDH levels, CD73 activity enables a good stratification between a favorable (CD73 low) and a poor survival group (CD73 high) (HR=2.94; 95\% CI 1.86 to $4.64 ; \mathrm{p}<0.0001$ ) (figure $5 \mathrm{c}$ ) but not in the patients' group with normal serum LDH levels. In patients with $B R A F$-mutated tumors, we observed that the OS is worse in patients who have both elevated levels of CD73 activity and $B R A F$ mutations versus patients with $B R A F$ mutations but low CD73 (HR=1.85; 95\% CI 1.11 to 3.08; $\mathrm{p}=0.02$ ) (figure 5d); no differences were observed in the patients' subgroup with BRAF wild-type tumors. We observed that the OS is worse in patients who have both elevated levels of $\mathrm{CD} 73$ and bone metastases (HR=2.41; 95\% CI 1.27 to $4.57 ; \mathrm{p}=0.006$ ) versus patients with bone metastases but low CD73 (figure 5e). A similar trend was observed in patients' subgroup with brain metastases and high CD73 versus patients with brain metastasis but low CD73 $(\mathrm{HR}=1.74 ; 95 \%$ CI 0.96 to $3015 ; \mathrm{p}=0.062)$ (figure $5 f)$.

\section{CD73 is prognostic in melanoma}

Finally, patients receiving nivolumab or pembrolizumab in monotherapy $(n=453)$ were analyzed by univariate and multivariate Cox regression analysis to evaluate potential associations of age, disease stage, sex, BRAF mutation status, serum LDH levels, line of treatment, brain or bone metastases and baseline serum CD73 activity with OS and 
Table 2 Cox regression analyses

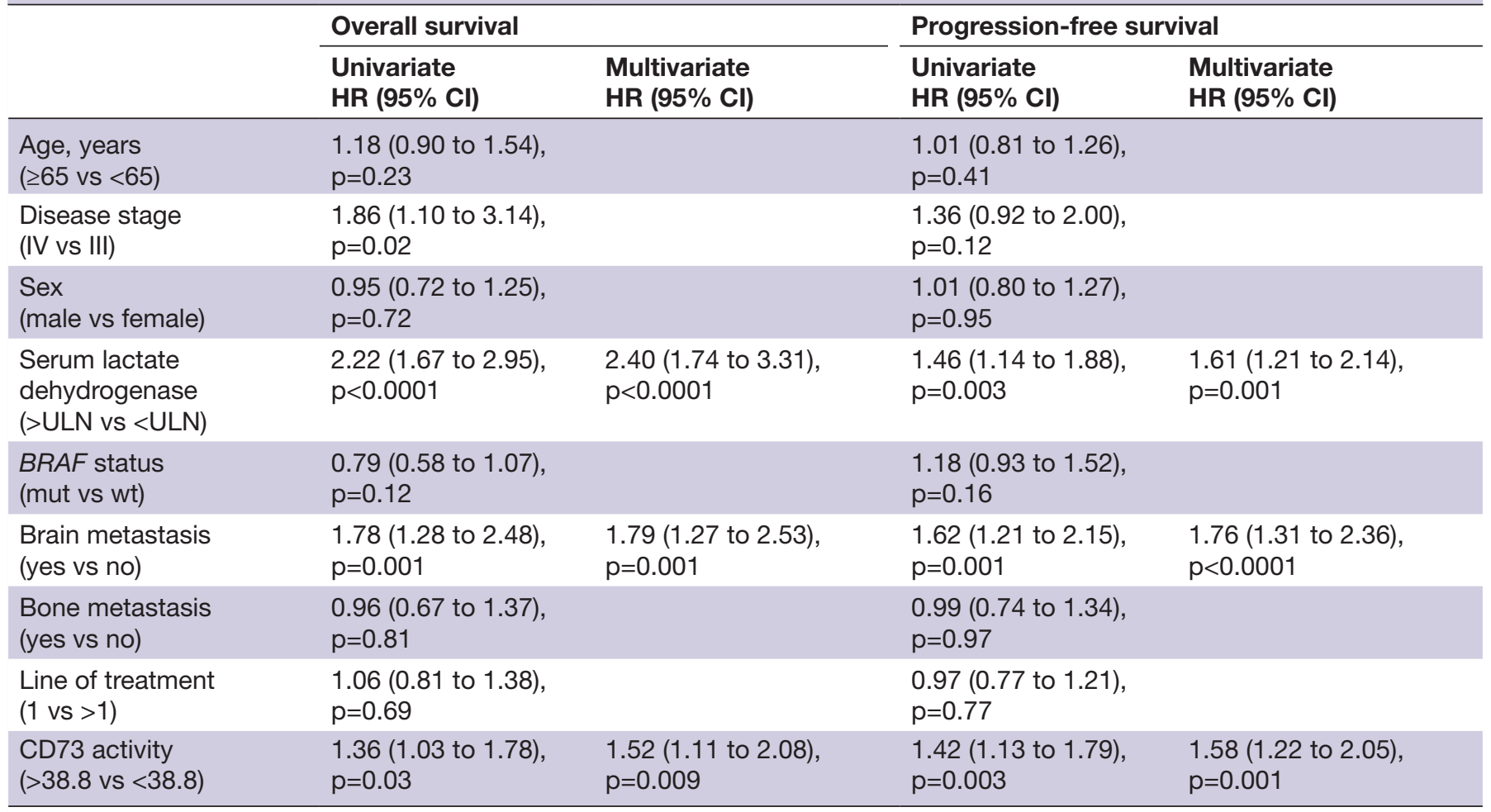

mut, mutated; ULN, upper limit of normal; wt, wild-type.

PFS. The univariate analysis showed that high levels of $\mathrm{LDH}$, the presence of brain metastases and high levels of serum CD73 enzymatic activity were significantly associated with a shorter OS and PFS (table 2). These factors were also analyzed in a multivariate model analysis: in addition to serum LDH and brain metastasis, serum CD73 activity appeared to be an independent prognostic factor (OS: HR=1.52, 95\% CI 1.11 to 2.08; $\mathrm{p}=0.009$; PFS: $\mathrm{HR}=1.5895 \%$ CI 1.22 to $2.05, \mathrm{p}=0.001$ ) (table 2).

\section{DISCUSSION}

In this study, we detected elevated levels of serum CD73 activity in patients with melanoma compared with healthy donors. Elevated baseline CD73 activity is strongly associated with worse response and survival in patients receiving anti-PD-1 therapy.

A number of studies have evaluated the potential prognostic value of high CD73 expression within tumor lesions, including lung, breast, gastric, ovarian cancer and metastatic colorectal cancer. ${ }^{12} 21$ In melanoma, CD73 is epigenetically regulated in malignant cells, and patients with melanoma show high frequency of the CD73 CpG island methylation in both primary and metastatic disease. ${ }^{22}$ Elevated tumor levels of CD73 have been found in patients with late-stage melanoma ${ }^{23}$ and high expression of CD73 in cells of metastatic lesions resulted associated with decreased OS. ${ }^{24}$ However, the relevance of CD73 expression in melanoma as prognostic factor is weak because of the heterogeneous expression of CD73 in primary melanoma versus metastatic melanoma. Moreover, expression of tumor CD73 can change in patients with melanoma with resistance to adoptive T cells transfer or anti-PD-1 therapy as well as in patients receiving BRAF/ MEK inhibitors. ${ }^{23} 25$

The soluble form of CD73 in the peripheral blood has been investigated in different types of cancer. Earlier studies in head and neck cancer demonstrated that the serum expression of CD73 was increased in patients with cancer compared with healthy subjects and its enzymatic activity was associated with the stage of cancer. ${ }^{26}$ Elevated blood levels of soluble CD73 were found in patients with colorectal cancer, prostate cancer and cervical cancer. ${ }^{27-29}$ Recently published data from patients with colorectal cancer liver metastases show that high levels of soluble CD73 expression are associated with shorter survival. ${ }^{21}$ These results indicate that soluble $\mathrm{CD} 73$ may serve as a non-invasive blood-based biomarker of cancer outcome. Yet, it is unclear whether soluble CD73 is a clinically useful biomarker of prognosis and response to immunotherapy in patients with melanoma. In the present study, we observed that patients with melanoma had elevated soluble CD73 in the peripheral blood and the enzymatic activity of CD73 well stratified patients with melanoma from healthy subjects. Elevated levels of CD73 enzymatic activity in peripheral blood may probably reflect an elevated expression of tissue CD73 expression. However, in this retrospective study we could not explore the level of CD73 in the tumor. Therefore, we do not know if there 
is any correlation between the circulating levels of soluble $\mathrm{CD} 73$ and those expressed in the tumor.

In melanoma, we previously proposed blood CD73 activity as biomarker of response to immunotherapy with nivolumab. ${ }^{20}$ The results of the present study further confirm these findings in a larger, multicentric and multinational, cohort of patients with melanoma and support the association between serum CD73 activity and clinical outcomes of immune checkpoint inhibitor therapy. Indeed, we observed that patients with high baseline levels of enzymatic CD73 activity presented a shorter OS than patients with low baseline CD73 activity on treatment with nivolumab or pembrolizumab. This result supports the notion that soluble CD73 activity in blood is a factor of poor prognosis in patients with melanoma that could complement the prognostic value of other features useful in directing patient therapy.

Among patients receiving anti-PD-1 monotherapy, we observed that patients with both high serum CD73 activity and high serum LDH presented an extremely poor prognosis. Similar results were obtained in patients with both high CD73 activity and bone metastasis or in patients with high CD73 activity and tumors with BRAF mutations. Although we did not find any significant difference in the baseline CD73 activity level in relationship with any clinicpathological characteristics of patients, a shorter OS was also found in patients $<65$ years and in females with high CD73 activity compared with low CD73 activity. Further investigation is needed to clarify the biological reason of these results. In the case of the patients' subgroups with brain or bone metastases or with high serum LDH, who have already poor survival outcomes, ${ }^{1}$ it is possible that the elevated levels of enzymatic activity of CD73 worsen the effectiveness of anti-PD-1 agents and reduced survival. Some gene alterations including BRAFand EGFR can drive the expression of CD73 in tumor cells. Moreover, BRAF inhibitors can reduce the expression of $\mathrm{CD} 73$ within tumor tissue. ${ }^{25}$ However, to the best of our acknowledge, there are no data on serum CD73 expression and activity in these patients' subgroup. This issue needs further investigations. Patients with cancer over the age of 60 years responded significantly better to treatment with the anti-PD-1 agents than younger patients in whom immune escape mechanisms are more likely to occur. ${ }^{30-32}$ Very recent data demonstrate that the expression of $\mathrm{CD} 73$ on $\mathrm{T}$ lymphocytes decreases in elderly healthy donors and in patients with head and neck squamous cell carcinoma, ${ }^{33}$ affecting to a lesser extent the response to treatment. Therefore, an elevated activity of CD73 may contribute to further impair the immune system response in younger patients, worsening the clinical outcomes to anti-PD-1 therapy.

Notably, the multivariate analysis from the patients' group treated with nivolumab or pembrolizumab confirms that the serum activity of CD73 was an independent predictor besides serum LDH levels, the presence of brain metastasis for both OS and PFS. These analyses were not performed in patients treated with nivolumab plus ipilimumab in combination, in whom the serum CD73 activity was not significantly associated with OS. It is to note that the HR values are very similar between the group treated with nivolumab or pembrolizumab alone (1.36) and the group treated with nivolumab plus ipilimumab (1.41). Most likely because of the small number of patients in the subgroup receiving nivolumab in combination with ipilimumab, the statistical significance is not reached. Therefore, we cannot make any reasonable conclusion and further analyses in a larger cohort of patients treated with anti-PD-1 monoclonal antibody in combination regimen are needed.

Baseline activity of CD73 resulted strongly associated with response to anti-PD-1 agents, especially in patients treated with anti-PD-1 agent alone as first line. In addition, in this latter group of patients in first-line therapy, PFS was significantly associated to serum CD73 activity. These data may suggest the potential relevance of CD73 as biomarker of response in patients treated with antiPD-1 agents specially as first-line setting. High-soluble CD73 activity may represent a mechanism of resistance to immunotherapy. Since CD73 is the main enzyme responsible of the production of extracellular adenosine, known to be a potent suppressor of T-cell-mediated antitumor immune response, our results raise the possibility that CD73-derived adenosine could counteract the antitumor immunity, limiting in turn the efficacy of anti-PD-1 agents. It is likely that this CD73-mediated mechanism of resistance would be relevant specially in patients treated first with anti-PD-1 agents in contrast to patients receiving these agents in second line applied after progression to prior line of therapy. Finally, we observed that after 3 months of treatment, available for this study, the CD73 activity did not change compared with baseline level and importantly, we noticed that non-responder patients still had higher levels of CD73 activity compared with responders. This suggest that the activity of $\mathrm{CD} 73$ in the peripheral blood did not change on-treatment, at least not between the two time points studied in this work. It is clear that further investigation is warranted to uncover the functional relevance of soluble $\mathrm{CD} 73$ in the peripheral blood as tumor immune evasion mechanism, during treatment with anti-PD-1 agents as well as in different regimen settings. In our opinion, these results reinforce the therapeutic potential of targeting CD73 in combination with anti-PD-1 agents to enhance responses and survival rates in patients with melanoma. Measurement of CD73 activity could be informative to identify a suppressive mechanism that influence the therapeutic effectiveness of immune checkpoint inhibitors and that regulate the antitumor immunity. Prospectively, CD73 activity may be useful in addition to prognostic parameters to guide therapeutic choices in patients with melanoma. In addition, measurement of CD73 may be also useful to identify patients who are likely to benefit from CD73-targeting agents that in combination with immunotherapy agents could further potentiate the clinical response. 


\section{CONCLUSIONS}

Our results suggest the possibility to use soluble CD73 as potential prognostic factor that could be added to other known markers for clinical outcomes in patients with unresectable melanoma.

\section{Author affiliations}

${ }^{1}$ Pharmacy, University of Salerno, Fisciano, Campania, Italy

${ }^{2}$ PhD Program in Drug Discovery and Development, University of Salerno, Fisciano, Campania, Italy

${ }^{3}$ Istituto Nazionale Tumori IRCCS Fondazione Pascale, Napoli, Campania, Italy

${ }^{4}$ Biostatistic Unit, Regina Elena Institute, Roma, Lazio, Italy

${ }^{5}$ Dermatology, University Hospital Zurich, Zurich, Switzerland

${ }^{6}$ Dermatology, Eberhard Karls Universitat Tubingen, Tubingen, Germany

${ }^{7} \mathrm{AP}$-HP Biochemistry, Hopital Saint-Louis, Paris, France

${ }^{8}$ Dermatology, University Hospital Essen, Essen, Nordrhein-Westfalen, Germany

${ }^{9}$ Soft Tissue/Bone Sarcoma and Melanoma, Maria Sklodowska-Curie Institute of

Oncology Warsaw, Warszawa, Poland

${ }^{10}$ APHP Dermatology, Diderot University, INSERM U976, Hôpital Saint-Louis, Paris, France

${ }^{11}$ UPMC Hillman Cancer Center, Pittsburgh, Pennsylvania, USA

${ }^{12}$ Comprehensive Cancer Center, University of Chicago, Chicago, IL, USA

\section{Twitter Teresa Amaral @TeresaSAmaral and Jason John Luke @jasonlukemd}

Contributors SM and PAA contributed to concept and design. All the authors contributed to acquisition, analysis or interpretation of data. RT and SM contributed to drafting of the manuscript. RT, DG, BW, TA, KW-H, PR, DS, CL, JJL, RD, AP, SM and PAA contributed to critical revision of the manuscript. DG, RT and SM contributed to statistical analysis. MCapone contributed to administrative, technical or material support. AP, SM and PAA contributed to study supervision.

Funding This work was supported by POR Campania FESR 2007-2013—0.0. 2.1-OCKEY (SM) and Italian Ministry of Health (IT-MOH) through "Ricerca Corrente" M2/M (PAA). GM has been founded by Istitutional "Ricerca Corrente". KWH and BW received funding from the Klaus Tschira Foundation (00.316.2017) for the Tübingen blood bank. KWH received funding for a 'junior research group' from the Medical Faculty of the University of Tübingen (2509-0-0).

Competing interests PAA has/had a consultant/advisory role for Bristol MyersSquibb, Roche-Genentech, Merck Sharp \& Dohme, Array, Novartis, Amgen, Merck Serono, Pierre Fabre, Incyte, NewLink Genetics, Genmab, Medimmune. He also received research funds from Bristol Myers-Squibb, Roche-Genentech, Array. KWH received commercial research grants from the Catalym $\mathrm{GmbH}$ and travel support from SITC (Society for Immunotherapy of Cancer). TA reports personal fees and travel grants from BMS, grants, personal fees and travel grants from Novartis, personal fees from Pierre Fabre, grants from Neracare, grants from Sanofi, outside the submitted work. RD has intermittent, project-focused consulting and/or advisory relationships with Novartis, Merck Sharp \& Dhome (MSD), Bristol-Myers Squibb (BMS), Roche, Amgen, Takeda, Pierre Fabre, Sun Pharma, Sanofi, Catalym, Second Genome, Regeneron, Alligator outside the submitted work. MPL intermittent project focused funding outside of the scope of the current work. LI intermittent project focused consulting and/or advisory relationship with Galderma outside the submitted work. DS reported consulting or advisory role for Roche/Genentech, Novartis, Bristol-Myers Squibb, MSD, Merck Serono, Amgen, Immunocore, Incyte, InFlarX, 4SC, Pierre Fabre, Mologen, Nektar, and Sanofi/Regeneron; honoraria from Roche/Genentech, Novartis, MSD, Bristol-Myers Squibb, Merck Serono, Amgen, Immunocore, Incyte, 4SC, Pierre Fabre, Array BioPharma, Pfizer, Philogen, and Regeneron; travel, accommodation and expenses from Roche/Genentech, Novartis, Bristol-Myers Squibb, Merck Serono, Amgen and Merck; speakers bureau for Novartis, Bristol-Myers Squibb, MSD, Pierre Fabre and Roche; research funding from Novartis and Bristol-Myers Squibb; steering committee member for Novartis, MSD and Bristol-Myers Squibb. PR has received honoraria for lectures and advisory boards from MSD, BMS, Novartis, Pierre Fabre, Amgen, Sanofi, Merck, Blueprint Medicines. JJL, Scientific Advisory Board: 7 Hills, Actym, Alphamab Oncology, Arch Oncology, Kanaph, Mavu, Onc. Al, Pyxis, Spring bank, Tempest; Consultancy: Abbvie, Algios, Array, Bayer, Bristol-Myers Squibb, Cstone, Eisai, EMD Serono, Janssen, Merck, Mersana, Novartis, PTx, RefleXion, Regeneron, Rubius, Silicon, Tesaro, Xilios; research support: (all to institution for clinical trials unless noted) AbbVie, Agios (IIT), Array (IIT), Astellas, Bristol-Myers Squibb, Corvus, EMD Serono, Immatics, Incyte, Kadmon, Macrogenics, Merck, Spring bank, Tizona, Xencor; travel: Bristol-Myers
Squibb, EMD Serono, Janssen, Merck, Mersana, Pyxis; patents: (both provisional) Serial \#15/612,657 (Cancer Immunotherapy), PCT/US18/36052 (Microbiome Biomarkers for Anti-PD-1/PD-L1 Responsiveness: Diagnostic, Prognostic and Therapeutic Uses Thereof). The other authors declare no potential competing interests.

\section{Patient consent for publication Not required.}

Ethics approval This study was approved by the Ethics Committee of Istituto Nazionale Tumori—IRCCS—Fondazione "G. Pascale", Naples, Italy (number 33/17 oss). A signed informed consent from patients was obtained for blood donation.

Data availability statement All data relevant to the study are included in the article or uploaded as supplementary information. N/A.

Supplemental material This content has been supplied by the author(s). It has not been vetted by BMJ Publishing Group Limited (BMJ) and may not have been peer-reviewed. Any opinions or recommendations discussed are solely those of the author(s) and are not endorsed by BMJ. BMJ disclaims all liability and responsibility arising from any reliance placed on the content. Where the content includes any translated material, BMJ does not warrant the accuracy and reliability of the translations (including but not limited to local regulations, clinical guidelines, terminology, drug names and drug dosages), and is not responsible for any error and/or omissions arising from translation and adaptation or otherwise.

Open access This is an open access article distributed in accordance with the Creative Commons Attribution Non Commercial (CC BY-NC 4.0) license, which permits others to distribute, remix, adapt, build upon this work non-commercially, and license their derivative works on different terms, provided the original work is properly cited, appropriate credit is given, any changes made indicated, and the use is non-commercial. See http://creativecommons.org/licenses/by-nc/4.0/.

\section{ORCID iDs}

Jason John Luke http://orcid.org/0000-0002-1182-4908

Kilian Wistuba-Hamprecht http://orcid.org/0000-0002-3104-8512

Silvana Morello http://orcid.org/0000-0002-6541-0997

\section{REFERENCES}

1 Larkin J, Chiarion-Sileni V, Gonzalez R, et al. Five-Year survival with combined nivolumab and ipilimumab in advanced melanoma. $N$ Engl J Med Overseas Ed 2019;381:1535-46.

2 Hamid O, Robert C, Daud A, et al. Five-Year survival outcomes for patients with advanced melanoma treated with pembrolizumab in KEYNOTE-001. Ann Oncol 2019;30:582-8.

3 Zimmermann $\mathrm{H}$, Zebisch M, Sträter N. Cellular function and molecular structure of ecto-nucleotidases. Purinergic Signal 2012;8:437-502.

4 Yegutkin GG. Nucleotide- and nucleoside-converting ectoenzymes: important modulators of purinergic signalling cascade. Biochim Biophys Acta 2008;1783:673-94.

5 Horenstein AL, Chillemi A, Zaccarello G, et al. A CD38/CD203a/ CD73 ectoenzymatic pathway independent of CD39 drives a novel adenosinergic loop in human $\mathrm{T}$ lymphocytes. Oncoimmunology 2013;2:e26246.

6 Stagg J, Smyth MJ. Extracellular adenosine triphosphate and adenosine in cancer. Oncogene 2010;29:5346-58.

7 Ohta A, Gorelik E, Prasad SJ, et al. A2A adenosine receptor protects tumors from antitumor T cells. Proc Natl Acad Sci U S A 2006;103:13132-7

8 Allard B, Pommey S, Smyth MJ, et al. Targeting CD73 enhances the antitumor activity of anti-PD-1 and anti-CTLA-4 mAbs. Clin Cancer Res 2013;19:5626-35.

9 lannone R, Miele L, Maiolino P, et al. Adenosine limits the therapeutic effectiveness of anti-CTLA4 mAb in a mouse melanoma model. Am J Cancer Res 2014;4:172-81.

10 Hay CM, Sult E, Huang Q, et al. Targeting CD73 in the tumor microenvironment with MEDI9447. Oncoimmunology 2016;5:e1208875.

11 Allard B, Allard D, Buisseret L, et al. The adenosine pathway in immuno-oncology. Nat Rev Clin Oncol 2020;17:611-29.

12 Jiang T, Xu X, Qiao M, et al. Comprehensive evaluation of NT5E/ CD73 expression and its prognostic significance in distinct types of cancers. BMC Cancer 2018;18:267.

13 Balch CM, Gershenwald JE, Soong S-J, et al. Final version of 2009 AJCC melanoma staging and classification. J Clin Oncol 2009;27:6199-206.

14 Eisenhauer EA, Therasse P, Bogaerts J, et al. New response evaluation criteria in solid tumours: revised RECIST guideline (version 1.1). Eur J Cancer 2009;45:228-47. 
15 Morello S, Turiello R, Madonna G, et al. Enzyme activity of circulating CD73 in human serum. Methods Enzymol 2019;629:257-67.

16 Goueli SA, Hsiao K. Monitoring and characterizing soluble and membrane-bound ectonucleotidases CD73 and CD39. PLoS One 2019;14:e0220094.

17 Pike AF, Kramer NI, Blaauboer BJ, et al. A novel hypothesis for an alkaline phosphatase 'rescue' mechanism in the hepatic acute phase immune response. Biochim Biophys Acta 2013;1832:2044-56.

18 Millán JL. Alkaline Phosphatases : Structure, substrate specificity and functional relatedness to other members of a large superfamily of enzymes. Purinergic Signal 2006;2:335-41.

19 Schneider E, Rissiek A, Winzer R, et al. Generation and function of Non-cell-bound CD73 in inflammation. Front Immunol 2019;10:10.

20 Morello S, Capone M, Sorrentino C, et al. Soluble CD73 as biomarker in patients with metastatic melanoma patients treated with nivolumab. J Trans/ Med 2017;15:244-44.

21 Messaoudi N, Cousineau I, Arslanian E, et al. Prognostic value of CD73 expression in resected colorectal cancer liver metastasis. Oncoimmunology 2020;9:1746138.

22 Wang H, Lee S, Nigro CL, et al. NT5E (CD73) is epigenetically regulated in malignant melanoma and associated with metastatic site specificity. Br J Cancer 2012;106:1446-52.

23 Young A, Ngiow SF, Madore J, et al. Targeting adenosine in BRAF-mutant melanoma reduces tumor growth and metastasis 2017;77:4684-96.

24 Monteiro I, Vigano S, Faouzi M, et al. Cd73 expression and clinical significance in human metastatic melanoma. Oncotarget 2018;9:26659-69.
25 Reinhardt J, Landsberg J, Schmid-Burgk JL, et al. Mapk signaling and inflammation link melanoma phenotype switching to induction of CD73 during immunotherapy. Cancer Res 2017;77:4697-709.

26 Lal H, Kumar L, Kohli GS, et al. Serum enzymes in head and neck cancer. IV: 5-nucleotidase. J Laryngol Otol 1989;103:200-2.

27 Huang Q, Durham NM, Sult E, et al. Abstract 1538: levels and enzyme activity of CD73 in primary samples from cancer patients 2015;75:1538-38.

28 Gardani CFF, Cappellari AR, de Souza JB, et al. Hydrolysis of ATP, ADP, and AMP is increased in blood plasma of prostate cancer patients. Purinergic Signal 2019;15:95-105.

29 de Lourdes Mora-García M, López-Cisneros S, Gutiérrez-Serrano V, et al. Hpv-16 infection is associated with a high content of CD39 and CD73 ectonucleotidases in cervical samples from patients with cin-1. Mediators Inflamm 2019;2019:1-13.

30 Wu Q, Wang Q, Tang X, et al. Correlation between patients' age and cancer immunotherapy efficacy. Oncoimmunology 2019;8:e1568810.

31 Kugel CH, Douglass SM, Webster MR, et al. Age correlates with response to anti-PD1, reflecting age-related differences in intratumoral effector and regulatory T-cell populations. Clin Cancer Res 2018;24:5347-56.

32 Li P, Yang X, Feng Y, et al. The impact of immunosenescence on the efficacy of immune checkpoint inhibitors in melanoma patients: a meta-analysis. Onco Targets Ther 2018;11:7521-7.

33 Jeske SS, Schuler PJ, Doescher J, et al. Age-Related changes in T lymphocytes of patients with head and neck squamous cell carcinoma. Immun Ageing 2020;17:3. 